\title{
Perfluorocarbon-perfused 23 gauge three-dimensional vitrectomy for complicated diabetic tractional retinal detachment
}

This article was published in the following Dove Press journal:

Clinical Ophthalmology

8 December 201I

Number of times this article has been viewed

\author{
Raul Velez-Montoya' \\ Jose Luis Guerrero- \\ Naranjo ${ }^{2}$ \\ Gerardo Garcia-Aguirre ${ }^{2}$ \\ Virgilio Morales-Cantón ${ }^{2}$ \\ Jans Fromow-Guerra ${ }^{2}$ \\ Hugo Quiroz-Mercado3 \\ 'Department of Ophthalmology, \\ University of Colorado Health and \\ Science Center, Rocky Mountain \\ Lions Eye Institute, University of \\ Colorado School of Medicine, CO, \\ USA; ${ }^{2}$ Retina Department, Asociación \\ para Evitar la Ceguera en México IAP, \\ México City, México; ${ }^{3}$ Department \\ of Ophthalmology, Denver Health \\ Medical Center, University of \\ Colorado School of Medicine, Denver, \\ CO, USA
}

Correspondence: Hugo Quiroz-Mercado Denver Health Medical Center,

777 Bannock Street,

Denver, CO 80204, USA

Email Hugo.Quiroz-Mercado@dhha.org
Background: Perfluorocarbon liquid (PCL)-perfused vitrectomy has been shown in previous studies to be feasible, safe, and to have advantages in managing complicated cases of tractional retinal detachment. The present study had the objectives of describing the anatomical results and measuring surgical time and PCL consumption when combining PCL-perfused techniques with modern vitrectomy equipment.

Methods: A prospective, interventional consecutive case series was investigated. We enrolled patients with diabetic tractional retinal detachment, complicated by proliferative vitreoretinopathy and poor vision. A 23 gauge PCL-perfused vitrectomy was done with three-dimensional settings. During the procedure, we assessed the degree of surgical bleeding, visualization quality, and difficulty of membrane dissections. Visual acuity, intraocular pressure, and anatomical success were assessed at one and 3 months of follow-up.

Results: Twelve patients were enrolled in this study. There were no statistical significant changes in intraocular pressure and visual acuity throughout the follow-up period. Surgery was performed in a hemorrhage-free environment in almost all cases, with good visualization and low technical difficulty. The mean complete surgical time was $94.92 \pm 25.03$ minutes. The mean effective vitrectomy time was $22.50 \pm 19.04$ minutes and the mean PCL consumption was $25.08 \pm 9.76 \mathrm{~mL}$, with a speed of $1.11 \mathrm{~mL} /$ minute. Anatomical success was $67 \%$ at 3 months. Conclusion: Although the technique proved to have some advantages in managing complicated cases of diabetic tractional retinal detachment, there was a high consumption of PCL. A redesign of the entire system is needed in order to decrease the amount of PCL needed for the technique.

Keywords: retinal detachment, diabetic, surgery, vitrectomy, vitreoretinopathy, perfluorocarbon

\section{Introduction}

Tractional retinal detachment is one of the major causes of severe visual loss in patients with proliferative diabetic retinopathy. ${ }^{1-3}$ In about $5 \%-10 \%$ of cases, proliferative vitreoretinopathy develops and complicates the anatomical and functional prognosis. ${ }^{4,5}$ Despite advances in surgical instrumentation and techniques, management of advanced cases of tractional retinal detachment, with or without proliferative vitreoretinopathy, continues to demand a mixture of surgical skill, experience, and good judgment. ${ }^{6,7}$

Vitrectomy techniques are very challenging in such cases. The vitreous body can be either transparent, making it difficult to identify and ensure its complete removal, or a dense vitreous hemorrhage can be encountered, precluding satisfactory visualization of intraocular structures, increasing the risk of iatrogenic damage. ${ }^{1,8}$ During surgery, particular attention is focused on minimizing intraoperative bleeding. ${ }^{1,9,10}$ 
The consequences of not doing so can range from poor visualization of the fundus (making the following surgical steps more difficult) to a complete failure of the surgery (uncontrollable bleeding). ${ }^{4,8,10}$ Usually, the formation of new clots generates "sticky" pseudomembranes that adhere very firmly to the healthy retina or to neovascular or fibrous proliferations, making it very hard to remove without doing retinotomies or causing more bleeding. However, the maneuver that probably demands more surgical expertise in complicated cases of tractional retinal detachment is dissection of the posterior hyaloid and fibrovascular membranes from attached or detached retina, because most of the time having a refined surgical technique is not sufficient. ${ }^{1,2,8}$ Surgeons must also have a complete and comprehensive understanding of the disease in order to succeed.

Since its introduction by Chang et al in 1987, the use of perfluorocarbon liquids (PCLs) as surgical adjuvants has spread throughout the world and currently forms part of every retina practice. ${ }^{11-14}$ PCLs are fluorinated carbon molecules, with a high affinity for oxygen. They are colorless and odorless substances with low viscosity. ${ }^{13}$ Their index of refraction is slightly different from that of saline, which allows good visibility. Perhaps their most important properties are their high density that helps flatten and stabilize the detached retina during surgery as well as their immiscibility with blood and other cellular debris. ${ }^{13-15}$

In a previous report, we described a surgical technique in which we used PCL as an infusion substance instead of regular saline. ${ }^{14}$ The advantages identified in this procedure were: its high oxygen-carrying capacity, which may be useful during surgery on an ischemic retina; oxygen affinity, which may facilitate elevation of intraocular pressure, allowing a hemorrhage-free surgery; immiscibility of PCL with blood and debris, which may facilitate surgery and improve surgical efficiency; and the high density of PCL, which may help to reattach and stabilize the retina during vitrectomy and membrane peeling. ${ }^{14,15}$ However, regardless of the aforementioned advantages, the original technique was described using vitrectomy probes of existing caliber (20 gauge) and available vitrectomy machines. Fluid control was extremely inefficient, and large losses of PCL during surgery were encountered. Therefore, a considerable amount of PCL was required to perform the surgery, transforming it into a less than cost-effective procedure. ${ }^{14}$ In a second report, we described the use of 25 gauge vitrectomy in order to minimize PCL loss during surgery, and although we were successful in reducing the amount of PCL required for the technique (up to $70 \%$ less) and had good anatomical results, the amounts were still too high. ${ }^{16}$
The present study has the aim of describing the feasibility and anatomical results of PCL perfused vitrectomy combined with dual dynamic drive software (three-dimensional, Alcon Laboratories, Fort Worth, TX) and 23 gauge vitrectomy with Eckardt valves in complicated cases of tractional retinal detachment, as well as measuring surgical time and comparing PCL consumption with previous studies by combining these technologies.

\section{Materials and methods}

A prospective, interventional consecutive case series was designed. The study was approved by the internal review board of the Asociación para Evitar la Ceguera en Mexico Hospital. The study was conducted according to the tenets of the Declaration of Helsinki. All patients signed an informed consent before any procedure was done and after a detailed explanation, according to the level of education of each individual patient, had been made. The study was conducted in the hospital facilities and was funded completely by the hospital research fund.

We enrolled treatment-naive patients (surgery) with a clinical diagnosis of longstanding diabetic tractional retinal detachment, complicated by proliferative vitreoretinopathy (grade C2 or higher, according to the Retina Society Classification $)^{17,18}$ and best-corrected visual acuity of 20/200 or worse, regardless of race, gender or previous retina photocoagulation, who rejected preoperative use of intravitreal bevacizumab despite a comprehensive explanation about its use. We excluded patients with previous filtration surgery, a clinical diagnosis of glaucoma, inflammatory eye disease, or with significant corneal, lens or other anterior chamber opacities that precluded fundus observations during follow-up.

All patients underwent a complete ophthalmological examination which included best-corrected visual acuity assessment using protocol Early Treatment Diabetic Retinopathy Study refraction, intraocular pressure measurement, and anterior chamber and fundus examination with indirect ophthalmoscopy. We followed the patients for a minimum of 3 months, and the follow-up appointments were at days 1, 30, and 90 after surgery. All measurements were repeated at every appointment.

\section{Surgical technique}

The main surgical technique used has been described elsewhere. ${ }^{14,15}$ The general tubing settings are depicted in Figure 1. The main differences are the following. A 23 gauge vitrectomy set was used, along with the Accurus 


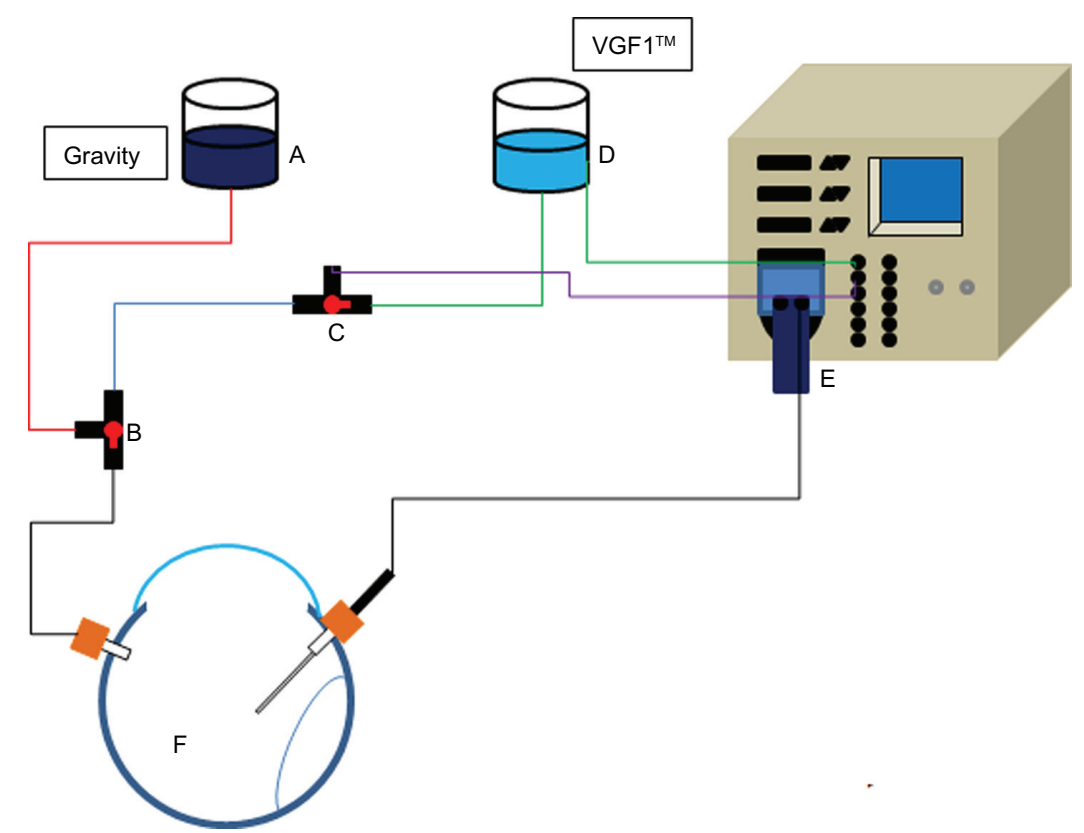

Figure I General diagram of PCL infusion system. (A) PCL container, (B and C) three-way valve, (D) saline container, (E) 23 gauge vitrectomy cassette, and (F) tractional retinal detachment.

Abbreviation: VGFI, Vented Gas Forced Infusion ${ }^{\mathrm{TM}}$ (Alcon Laboratory, Fort Worth, TX).

Surgical System and XS4 fourth-generation software, threedimensional technology (Alcon Laboratories) and an Eckard valve (Dutch Ophthalmic Research Center, Scheijdelveweg, Zuidland, The Netherlands) which has plastic tabs to prevent fluid loss during surgery. None of the patients received intravitreal bevacizumab prior to surgery. The surgical parameters were adjusted according to surgeon preference (HQM). The cuts per minute ranged between 2500 and 1500; the vacuum level was selected according to each individual case. The intraocular infusion pressure ranged between 25 to $30 \mathrm{mmHg}$. The selection of surgical tamponade was done according to each case in particular. The assessment of surgical bleeding and visualization quality was done by a third person (not related to the surgical procedure) by reviewing the digital videos of the surgery and graded according to subjective scales. Surgical bleeding was classified according to its magnitude, ie, mild (did not prevent visualization and was easy to aspirate), moderate (prevented visualization for small periods of time but was still easy to aspirate), and severe (prevented visualization completely, blood located in the interface between PCL and balanced salt solution, and was hard or impossible to aspirate). The quality of retina visualization during surgery was classified according to a subjective system of five levels, ie, very good (if retinal fundus and its capillary vasculature could be identified), good (if retina fundus but only main retinal vasculature could be identified), fair (if only vascular proliferations and optic nerve, but no retina fundus could be identified), bad (if only the optic nerve or some proliferations could be identified), and very bad (if one could not identify any structure). The difficulty of the dissection of posterior hyaloid and fibrovascular membranes was graded by the surgeon himself at the end of the surgery. Quantification of the amount of PCL used during the procedure was done by the second surgeon via recollecting the PCL from the vitrectomy cassette bag. The complete surgical time (from anesthesia to the placement of the eye patch) and the effective vitrectomy time (from insertion of vitrectomy to end of vitreous removal) was drawn from the Accurus timer. Effective vitrectomy time included time spent on dissection of the posterior hyaloid but not time spent on dissecting fibrovascular membranes or vitreoretinal proliferation peeling.

The PCL used was perfluoro-N-octane $\left(\mathrm{C}_{3} \mathrm{~F}_{18}\right)$ with a viscosity of $0.8 \mathrm{CsT}$, refractive index of 1.27 , and specific gravity of 1.76. PCL was purified and filtered under sterile conditions with a Millipore filter of $0.45 \mu \mathrm{m}$ and $0.2 \mu \mathrm{m}$ (Bedford, MA) and sterilized with ethylene oxide and placed in sterile containers. This form of PCL was not commercially available at that time but was supplied with a medical pureness degree by a private manufacturer (Vitreoretinal Technologies, Irving CA).

Statistical analysis was performed using SPSS software version 10.1.0 (Sigma Stat; Systat Software Inc, San Jose, CA). Data are presented as the median and standard 
deviation. For data analysis, a two-tailed $t$-test was used. A $P$ value $<0.05$ was considered statistically significant.

\section{Results}

A total of 12 patients (seven females, 58.3\%) were enrolled in the study. The demographic data are summarized in Table 1. The median age of the group was $52.25 \pm 8.6$ years. Of the 12 patients, nine $(75 \%)$ had the macula off at the time of enrollment. The median time between visual loss and retinal surgery was $9.2 \pm 2.5$ (range 4-12) months. Most of the patients had $\mathrm{C} 2$ or $\mathrm{C} 3$ vitreoretinal proliferation; only two had C4 vitreoretinal proliferation (patients 2 and 11). The mean intraocular pressure at baseline was $14.54 \pm 2.5 \mathrm{mmHg}$, $18.5 \pm 2.7 \mathrm{mmHg}$ at one month, and $15.71 \pm 1.8 \mathrm{mmHg}$ at 3 months of follow-up. There was no statistical difference regarding intraocular pressure. A summary of best-corrected visual acuity throughout the follow-up period is depicted in Table 1, and although there was a trend of improvement, there were no statistical differences $(P=0.07$ at one month and $P=0.06$ at 3 months).

Surgical characteristics regarding severity of hemorrhage, visual quality, and difficulty of membrane peeling are summarized in Table 2. Most of the surgeries were performed in a hemorrhage-free environment (75\%). Only three of them encountered moderate bleeding during surgery. Visual quality was labeled from "good" to "very good", and the surgeon described the difficulty of membrane and posterior hyaloid peeling as easy to moderate.

Surgical times and PCL consumption are summarized in Table 3. Mean complete surgical time was $94.92 \pm 25.03$ (range 54-145) minutes. Mean effective vitrectomy time was

Table I Proliferative vitreoretinopathy classification, according to the Retina Society Classification

\begin{tabular}{clllllll}
\hline No & Age & Gender & Macula & $\begin{array}{l}\text { PVR } \\
\text { Baseline } \\
\text { BCVA }\end{array}$ & $\begin{array}{l}\text { BCVA } \\
\text { I mo }\end{array}$ & $\begin{array}{l}\text { BCVA } \\
\text { 3mo }\end{array}$ \\
\hline I & 48 & M & OFF & C2,3 & CF I m & $20 / 800$ & $20 / 800$ \\
2 & 54 & F & OFF & C2,4 & HM & CF I m & $20 /$ I000 \\
3 & 29 & F & OFF & C3 & HM & HM & HM \\
4 & 55 & M & OFF & C2,3 & CF I m & CF I m & CF I m \\
5 & 49 & M & ON & C2,3 & $20 / 400$ & $20 / 400$ & $20 / 320$ \\
6 & 53 & F & OFF & C4 & CF 4 m & CF I m & CF I m \\
7 & 57 & F & ON & C2 & $20 / 320$ & $20 / 320$ & $20 / 200$ \\
8 & 48 & M & OFF & C3 & HM & $20 / 800$ & $20 / 320$ \\
9 & 55 & M & OFF & C2 & CF I m & $20 / 800$ & $20 / 630$ \\
I0 & 62 & F & OFF & C3 & CF I m & $20 / 1000$ & $20 / 800$ \\
II & 56 & F & OFF & C3,4 & CF I m & CF I m & $20 / 800$ \\
I2 & $6 I I$ & F & ON & C3 & $20 / 200$ & $20 / 200$ & $20 / 400$ \\
\hline A6 & & &
\end{tabular}

Abbreviations: BCVA, best-corrected visual acuity; CF I $\mathrm{m}$, count fingers at I meter; HM, hand movement; $M$, male; F, female.
Table 2 Subjective surgery measurements

\begin{tabular}{clll}
\hline No & Hemorrhage & Visual quality & Membrane peeling \\
\hline I & Moderated & VG & Moderated \\
2 & Mild & VG & Moderated \\
3 & Mild & VG & Easy \\
4 & Moderated & G & Hard \\
5 & Mild & G & Easy \\
6 & Mild & G & Easy \\
7 & Mild & VG & Easy \\
8 & Mild & VG & Moderated \\
9 & Mild & G & Easy \\
10 & Mild & VG & Ease \\
II & Mild & VG & Moderated \\
I2 & Moderated & VG & Ease \\
\hline
\end{tabular}

Abbreviations: VG, very good; G, good.

$22.50 \pm 19.04$ (range 11-80) minutes. Mean PCL consumption was $25.08 \pm 9.76 \mathrm{~mL}$, with an average consumption per minute of $1.11 \mathrm{~mL}$ of PCL. Silicon oil (5000 CsT) was used as surgical tamponade in all cases. Anatomical success was obtained in all cases after surgery but, after 3 months of follow-up, only eight patients (67\%) had an attached retina. Of the remaining cases, two had small inferior detachment and were treated successfully with laser photocoagulation. The other two had significant inferior and superior detachments due to reproliferation of vitreoretinal proliferation and required a second vitrectomy combined with a scleral buckle. Anatomical success was obtained in both cases after the second surgery. There were no complications related to the surgical technique.

\section{Discussion}

Since its introduction by Machemer in 1970, the indications for vitrectomy have been expanded tremendously and today it is one of the most commonly performed eye surgeries. ${ }^{19,20}$

Table 3 Surgical times

\begin{tabular}{rlll}
\hline No & CST (min) & EVT $(\mathbf{m i n})$ & PCL $(\mathbf{m L})$ \\
\hline 1 & 92 & 31 & 30 \\
2 & 70 & 16 & 32 \\
3 & 54 & 12 & 23 \\
4 & 130 & 22 & 45 \\
5 & 85 & 18 & 8 \\
6 & 97 & 12 & 18 \\
7 & 116 & 24 & 21 \\
8 & 145 & 80 & 35 \\
9 & 80 & 14 & 18 \\
10 & 92 & 11 & 20 \\
11 & 90 & 17 & 30 \\
12 & 88 & 13 & 21 \\
\hline
\end{tabular}

Abbreviations: CST, complete surgery time; EVT, effective vitrectomy time; PCL, perfluorocarbon liquid consumption. 
Its applications include vitreous hemorrhages, retinal detachment, macular edema, macular holes, and vitreoretinal interface abnormalities, among others. ${ }^{21-24}$ The adoption of PCL as a surgical tool has proved to be safe and nontoxic to the retina, even in cases of accidental retained droplets after vitrectomy or while used as a short-term tamponade. ${ }^{14,25,26}$ In the present study, replacement of balanced salt solution with PCF as the infusion substance appears to be safe and helped with resolution of cases. The special physical properties of PCF allowed quick stabilization of the retina in a hemorrhage-free environment as well as quick reattachment while maintaining excellent visualization of the intraocular structures.

However, although there was improved fluid control, better surgical equipment, and updated software, the total amount of PCL consumed was still high and almost identical to that previously reported in the 25 gauge study $(24.38 \mathrm{~mL}$ versus $25.08 \mathrm{~mL}$ ). The speed of consumption was also very fast $(1.11 \mathrm{~mL} /$ minute). Therefore, the cost-effectiveness of the procedure is still very poor. During review of the system, we became aware that most of the PCL used is wasted on tubing and dead spaces in the system. Thus, the authors speculate that if they could design a system with a significant reduction in the length of the infusion line along with a specially designed bottle and recirculation device (which could separate cellular debris from PCL and perhaps oxygenate it at the same time), we could effectively decrease the amount of PCL needed for the technique and reduce the speed of consumption by up to $0.3 \mathrm{~mL} /$ minute. Perhaps then the cost of doing the surgery will be no obstacle to performing it more often and prospective studies with larger sample sizes and larger follow-up periods could be conducted.

Another option for reducing bleeding during vitrectomy is the use of intravitreal bevacizumab prior to surgery. ${ }^{1,27}$ Studies have shown that a previous dose can decrease not only bleeding, but also the need of endodiathermy, need for frequent surgical tool exchange, and operative time, while increasing the number of blunt dissections against sharp dissections, which is indirect evidence of a more straightforward procedure. ${ }^{1,27}$ The combination of intravitreal bevacizumab prior to surgery with a PCL-perfused technique could pose greater benefits than both techniques used separately. In this study, candidates were selected from patients who had actively refused use of preoperative bevacizumab after an explanation of possible cardiovascular and cerebrovascular side effects. As an alternative way to prevent massive bleeding during the procedure, we offered the surgical technique described above. Therefore, the low numbers of patients enrolled in this study could be explained by the rarity of the pathology and the reduced number of available patients for consent.

In two recent reports, Arevalo described a variation of this technique and named it "en bloc perfluorodissection". ${ }^{28,29}$ In these reports, Arevalo acknowledged the special advantages of the technique and highlighted the capacity of PCL for dissecting the posterior hyaloid from fibrovascular membranes. He also concurred about the improved visualization of vitreous and intraocular structures, the rapid retinal reattachment and quick resolution of subretinal fluid during surgery, and easier dissection of epiretinal membranes and posterior hyaloid in a PCL-filled vitreous cavity. ${ }^{28,29}$ An evident advantage of en bloc perfluorodissection over PCF-perfused vitrectomy would be the small amount of PCL required for en bloc perfluorodissection (mean $4 \mathrm{~mL}$, range 3-8 $\mathrm{mL}$ ). The author also expressed concerns about PCL pressing on the posterior hyaloid and fibrovascular tissue, increasing the degree of difficulty of its dissection. ${ }^{28,29}$ However, even though we noticed posterior displacement of such structures, the vacuum of the vitrectomy probe and the force of the grip from the surgical forceps have not caused any problems in handling the posterior hyaloid, and the maneuver is still labeled as easy by the surgeon. This could vary according to the surgical skills of each surgeon; therefore, appropriate selection of cases should be done according to individual level of expertise.

Complicated diabetic tractional retinal detachment, with or without proliferative vitreoretinopathy, is known to be one of the most challenging retinal surgeries. ${ }^{19,30}$ The anatomical prognosis is poor and usually accompanied by a poor visual prognosis due to macular involvement and extremely ischemic retinas. ${ }^{1}$ Nevertheless, numerous recent studies have documented the benefits of surgical treatment of diabetic retinopathy involving the macula. ${ }^{19,31,32}$ Visual improvement has been reported in up to $75 \%$ of cases, with anatomical success ranging from $66 \%$ to $88 \% .^{19,31,32}$ Moreover, trials like the Diabetic Retinopathy Vitrectomy Study have found that not doing surgery can lead to severe visual loss $(<5 / 200)$ in up to $15 \%$ of cases after one year and $23 \%$ after 2 years. ${ }^{33,34}$ Additionally, several large clinical trials have demonstrated that certain ethnic groups (Blacks and Latinos) have an increased incidence of type 2 diabetes. ${ }^{35,36}$ The Los Angeles Latino Eye Study reported that being Latino, especially with Mexican ascendance, poses an increased risk for severe, vision-threatening diabetic retinopathy, including proliferative stages. ${ }^{35,37}$ In this study, all the tamponades were made with 5000 CsT silicon oil. Although the surgeon had other choices, due to the severity and complexity of each particular case the surgeon considered the use of this silicon oil as a more appropriate tamponade method. 
In the present study, although anatomical success was obtained in all cases after surgery, only $67 \%$ remained attached after 3 months of follow-up. Statistical analysis of the best-corrected visual acuity showed a trend towards improvement but the change was not statistically significant. The poor anatomical and visual outcomes could be explained by the severity of all cases along with the ethnicity of the sample and incidence of longstanding disease. During follow-up, most of the patients in whom the retina reattached successfully presented very pale retinas and various degrees of macular ischemia (data not shown).

PCL is commonly used as a tool in the surgical management of complicated retinal detachments and proliferative vitreoretinopathy. ${ }^{38}$ It is easily available, easy to use, and has few complications. ${ }^{38,39}$ The main undesired effect is unintended retention either in the preretinal space (anterior chamber and vitreous cavity) or in the subretinal space. ${ }^{39}$ Although there are studies that prove the safety of longstanding intraocular retention, there are concerns that prolonged retention may cause underlying retinal pigment epithelium atrophy, subretinal fibrosis, and scotomas. ${ }^{25,26,38,40,41}$ The main risk factor for unintended retention seems to be large peripheral retinotomies and the absence of saline rinse after its removal. ${ }^{39}$ Posterior small-sized and medium-sized retinal breaks do not seem to increase the risk of subretinal retention of PCL. However, this technique was designed for patients with significant traction in the posterior pole and periphery, which may in turn increase the risk for subretinal migration of PCL through these retinal defects, especially during initial instillation of PCL in which small-sized and medium-sized droplets may form in the posterior pole. Nevertheless, after all the droplets coalesce into a single big bubble, its superficial tension will prevent passage through small retinal defects, even despite the presence of significant traction. Either way, a careful selection and extensive preoperative examination of every particular case is strongly advised.

This study has some weaknesses in addition to the small sample number and short follow-up time, which the authors would like to address. First, most of the surgical variables were measured subjectively, which may create a bias. A more appropriate approach would have been the quantification of indirect markers, like the number of times endodiathermy was needed (in the case of qualifying bleeding), as well as application of validated scales for measuring difficulty, like the one published by Grigorian et al, in order to have a more uniform group in terms of surgical difficulty. ${ }^{42}$ The quantification of surgical bleeding could have been done also by examining the vitrectomy cassette. As a matter of fact, the original study design contemplated examination of the surgical cassette as a way to assess bleeding. However, during the study, we realized that some patients shared the same surgical cassette, making analysis impossible in this way and forcing us to use more subjective ways to assess bleeding. Second, assessment of difficulty of posterior hyaloid and membrane peeling was done directly by the surgeon. The subjective experience could vary depending on the degree of expertise of the surgeon and cannot be taken as an absolute measurement. Finally, there was an obvious selection bias in recruiting patients.

In summary, perfluorocarbon-perfused vitrectomy with 23 gauge caliber and three-dimensional software is feasible, safe, and could help in managing difficult cases of advanced diabetic retinal detachments with excellent quality of visualization and a hemorrhage-free environment. The physical properties of PCL can help in dissection of critical structures like the posterior hyaloid and fibrovascular membranes. Despite the strengths and advantages of the technique, the high amount of PCL needed for the realization of surgery prevents its routine use and, unless necessary modifications are made to the system, performance of further studies regarding this technique will be difficult.

\section{Acknowledgment}

The authors thank Melanie Soberon, College of Agriculture and Life Sciences, Cornell University, New York, for her help in the editing process.

\section{Disclosure}

A preliminary version of this study was presented at the Jules Gonin meeting 2006 (Cape Town, South Africa) and as a poster presentation at the Association for Research in Vision and Ophthalmology annual meeting in 2007 at Fort Lauderdale, FL. This report was fully sponsored and conducted by the Asociación para Evitar la Ceguera Research Fund, in México City.

\section{References}

1. da R Lucena D, Ribeiro JA, Costa RA, et al. Intraoperative bleeding during vitrectomy for diabetic tractional retinal detachment with versus without preoperative intravitreal bevacizumab (IBeTra study). $\mathrm{Br} J$ Ophthalmol. 2009;93(5):688-691.

2. Rice TA, Michels RG, Rice EF. Vitrectomy for diabetic traction retinal detachment involving the macula. Am J Ophthalmol. 1983;95(1):22-33.

3. Fong DS, Ferris FL 3rd, Davis MD, Chew EY. Causes of severe visual loss in the early treatment diabetic retinopathy study: ETDRS report no. 24. Early Treatment Diabetic Retinopathy Study Research Group. Am J Ophthalmol. 1999;127(2):137-141.

4. Regler R, Sachs HG, Hillenkamp J, Helbig H, Framme C. Long-term evaluation of anatomic and functional results after complicated retinal detachment treated with pars plana vitrectomy and heavy silicone oil tamponade. Klin Monbl Augenheilkd. 2009;226(9):707-712. 
5. Kirchhof B. Strategies to influence PVR development. Graefes Arch Clin Exp Ophthalmol. 2004;242(8):699-703.

6. Maia OO Jr, Takahashi WY, Helal Junior J. Triamcinolone-assisted 3D-vitrectomy in diabetic vitreomacular traction. Arq Bras Oftalmol. 2008;71(4):518-522.

7. Yamakiri K, Sakamoto T, Noda Y, et al. Reduced incidence of intraoperative complications in a multicenter controlled clinical trial of triamcinolone in vitrectomy. Ophthalmology. 2007;114(2):289-296.

8. Ho T, Smiddy WE, Flynn HW Jr. Vitrectomy in the management of diabetic eye disease. Surv Ophthalmol. 1992;37(3):190-202.

9. Oshima Y, Shima C, Wakabayashi T, et al. Microincision vitrectomy surgery and intravitreal bevacizumab as a surgical adjunct to treat diabetic traction retinal detachment. Ophthalmology. 2009;116(5):927-938.

10. Bartz-Schmidt KU, Kirchhof B, Heimann K. Primary vitrectomy for pseudophakic retinal detachment. Br J Ophthalmol. 1996;80(4):346-349.

11. Chang S. Low viscosity liquid fluorochemicals in vitreous surgery. $A m$ J Ophthalmol. 1987;103(1):38-43.

12. Chang S, Ozmert E, Zimmerman NJ. Intraoperative perfluorocarbon liquids in the management of proliferative vitreoretinopathy. $\mathrm{Am} \mathrm{J}$ Ophthalmol. 1988;106(6):668-674.

13. Imamura Y, Minami M, Ueki M, Satoh B, Ikeda T. Use of perfluorocarbon liquid during vitrectomy for severe proliferative diabetic retinopathy. Br J Ophthalmol. 2003;87(5):563-566.

14. Quiroz-Mercado H, Guerrero-Naranjo J, Agurto-Rivera R, et al. Perfluorocarbon-perfused vitrectomy: a new method for vitrectomy - a safety and feasibility study. Graefes Arch Clin Exp Ophthalmol. 2005;243(6):551-562.

15. Quiroz-Mercado H, Suarez-Tata L, Magdalenic R, et al. Perfluorocarbon perfused vitrectomy: animal studies. Am J Ophthalmol. 2004; 137(2):287-293.

16. Quiroz-Mercado H, Garcia-Aguirre G, Ustariz-Gonzalez O, MartinAvia J, Martinez-Jardon S. Perfluorocarbon-perfused vitrectomy using a transconjunctival 25-gauge system. Retina. 2007;27(7):926-931.

17. Rizzo S, Romagnoli MC, Genovesi-Ebert F, Belting C. Surgical results of heavy silicone oil HWS-45 3000 as internal tamponade for inferior retinal detachment with PVR: a pilot study. Graefes Arch Clin Exp Ophthalmol. 2011;249(3):361-367.

18. Machemer R, Aaberg TM, Freeman HM, Irvine AR, Lean JS, Michels RM. An updated classification of retinal detachment with proliferative vitreoretinopathy. Am J Ophthalmol. 1991;112(2):159-165.

19. Eliott D, Hemeida T. Diabetic traction retinal detachment. Int Ophthalmol Clin. 2009;49(2):153-165.

20. Machemer R. The development of pars plana vitrectomy: a personal account. Graefes Arch Clin Exp Ophthalmol. 1995;233(8):453-468.

21. Tao Y, Jiang YR, Li XX, Gao L, Jonas JB. Long-term results of vitrectomy without endotamponade in proliferative diabetic retinopathy with tractional retinal detachment. Retina. 2010;30(3):447-451.

22. Aras $\mathrm{C}$, Arici $\mathrm{C}, \mathrm{Akar} \mathrm{S}$, et al. Peeling of internal limiting membrane during vitrectomy for complicated retinal detachment prevents epimacular membrane formation. Graefes Arch Clin Exp Ophthalmol. 2009;247(5):619-623.

23. Aaberg TM, Abrams GW. Changing indications and techniques for vitrectomy in management of complications of diabetic retinopathy. Ophthalmology. 1987;94(7):775-779.

24. Harbour JW, Smiddy WE, Flynn HW Jr, Rubsamen PE. Vitrectomy for diabetic macular edema associated with a thickened and taut posterior hyaloid membrane. Am J Ophthalmol. 1996;121(4):405-413.

Clinical Ophthalmology

\section{Publish your work in this journal}

Clinical Ophthalmology is an international, peer-reviewed journal covering all subspecialties within ophthalmology. Key topics include: Optometry; Visual science; Pharmacology and drug therapy in eye diseases; Basic Sciences; Primary and Secondary eye care; Patient Safety and Quality of Care Improvements. This journal is indexed on Submit your manuscript here: http://www.dovepress.com/clinical-ophthalmology-journal
25. Velikay M, Wedrich A, Stolba U, Datlinger P, Li Y, Binder S. Experimental long-term vitreous replacement with purified and nonpurified perfluorodecalin. Am J Ophthalmol. 1993;116(5):565-570.

26. Schatz B, El-Shabrawi Y, Haas A, Langmann G. Adverse side effects with perfluorohexyloctane as a long-term tamponade agent in complicated vitreoretinal surgery. Retina. 2004;24(4):567-573.

27. Rizzo S, Genovesi-Ebert F, Di Bartolo E, Vento A, Miniaci S, Williams G. Injection of intravitreal bevacizumab (Avastin) as a preoperative adjunct before vitrectomy surgery in the treatment of severe proliferative diabetic retinopathy (PDR). Graefes Arch Clin Exp Ophthalmol. 2008;246(6):837-842.

28. Arevalo JF. En bloc perfluorodissection in vitreoretinal surgery: a new surgical technique. Retina. 2008;28(4):653-656.

29. Arevalo JF. En bloc perfluorodissection for tractional retinal detachment in proliferative diabetic retinopathy. Ophthalmology. 2008;115(6):e21-e25.

30. Shah CP, Ho AC, Regillo CD, Fineman MS, Vander JF, Brown GC. Short-term outcomes of 25-gauge vitrectomy with silicone oil for repair of complicated retinal detachment. Retina. 2008;28(5):723-728.

31. Douglas MJ, Scott IU, Flynn HW Jr. Pars plana lensectomy, pars plana vitrectomy, and silicone oil tamponade as initial management of cataract and combined traction/rhegmatogenous retinal detachment involving the macula associated with severe proliferative diabetic retinopathy. Ophthalmic Surg Lasers Imaging. 2003;34(4):270-278.

32. Williams DF, Williams GA, Hartz A, Mieler WF, Abrams GW, Aaberg TM. Results of vitrectomy for diabetic traction retinal detachments using the en bloc excision technique. Ophthalmology. 1989;96(6):752-758.

33. [No authors listed]. Two-year course of visual acuity in severe proliferative diabetic retinopathy with conventional management. Diabetic Retinopathy Vitrectomy Study (DRVS) report \#1. Ophthalmology. 1985;92(4):492-502.

34. [No authors listed]. Early vitrectomy for severe vitreous hemorrhage in diabetic retinopathy. Two-year results of a randomized trial. Diabetic Retinopathy Vitrectomy Study report 2. The Diabetic Retinopathy Vitrectomy Study Research Group. Arch Ophthalmol. 1985;103(11): 1644-1652.

35. Flaxel CJ, Dustin L, Kim J, Bekendam P, Row P. Outcome of diabetic vitrectomy in Latino population. Retina. 2007;27(9):1274-1278.

36. Davidson MB. The disproportionate burden of diabetes in AfricanAmerican and Hispanic populations. Ethn Dis. 2001;11(1):148-151.

37. Varma R, Torres M, Pena F, Klein R, Azen SP. Prevalence of diabetic retinopathy in adult Latinos: the Los Angeles Latino eye study. Ophthalmology. 2004;111(7):1298-1306.

38. Tewari A, Eliott D, Singh CN, Garcia-Valenzuela E, Ito Y, Abrams GW. Changes in retinal sensitivity from retained subretinal perfluorocarbon liquid. Retina. 2009;29(2):248-250.

39. Garcia-Valenzuela E, Ito Y, Abrams GW. Risk factors for retention of subretinal perfluorocarbon liquid in vitreoretinal surgery. Retina. 2004; 24(5):746-752.

40. Blinder KJ, Peyman GA, Desai UR, Nelson NC Jr, Alturki W, Paris CL. Vitreon, a short-term vitreoretinal tamponade. $\mathrm{Br} J$ Ophthalmol. 1992;76(9):525-528.

41. Lee GA, Finnegan SJ, Bourke RD. Subretinal perfluorodecalin toxicity. Aust N Z J Ophthalmol. 1998;26(1):57-60.

42. Grigorian RA, Castellarin A, Fegan R, et al. Epiretinal membrane removal in diabetic eyes: comparison of viscodissection with conventional methods of membrane peeling. Br J Ophthalmol. 2003;87(6):737-741.

\section{Dovepress}

PubMed Central and CAS, and is the official journal of The Society of Clinical Ophthalmology (SCO). The manuscript management system is completely online and includes a very quick and fair peer-review system, which is all easy to use. Visit http://www.dovepress.com/ testimonials.php to read real quotes from published authors. 